\title{
Previous Intestinal Resection Is Associated with Postoperative Complications in Crohn's Disease: A Cohort Study
}

\author{
Yantao Duan, Yifan Liu, and Yousheng Li \\ Department of General Surgery, Shanghai Ninth People's Hospital, Shanghai Jiao Tong University School of Medicine, \\ Shanghai, China \\ Correspondence should be addressed to Yousheng Li; 1306530916@qq.com
}

Received 7 July 2020; Revised 1 September 2020; Accepted 9 September 2020; Published 15 September 2020

Academic Editor: Fariborz Mansour-ghanaei Copyright $\odot 2020$ Yantao Duan et al. This is an open access article distributed under the Creative Commons Attribution License,
which permits unrestricted use, distribution, and reproduction in any medium, provided the original work is properly cited.

\begin{abstract}
Background. To assess the influence of a previous intestinal resection on postoperative complications for Crohn's disease (CD). Methods. Data on patients with CD undergoing surgery in our department from January 2016 through December 2019 were retrospectively reviewed. Information collected included demographic details, surgical data, and postoperative outcome. A crosssectional study design was employed. Associations between postoperative complications and preoperative clinical indicators were further analyzed. Results. Of the 129 patients with CD studied, 62 patients (48.06\%) underwent previous resection. These patients were more likely to be older $(P=0.031)$, have longer disease duration $(P=0.025)$, use less 5-aminosalicylic acid/sulfasalazine preoperatively $(P=0.013)$, have lower body mass index $(P=0.003)$, and have a higher American Society of Anesthesiologists (ASA) Physical Status Classification System score $(P=0.043)$. Patients who had previous surgery had a longer duration of operation $(P=0.003)$, greater estimated blood loss $(P=0.001)$, and longer hospital stay $(P<0.001)$ and were more inclined to develop postoperative complications $(P=0.047)$, particularly anastomotic leak $(P=0.021)$ and severe $(C l a v i e n-$ Dindo grade III/IV) complications $(P=0.038)$. After multivariate analysis, previous intestinal resection $(P=0.019)$, preoperative use of steroids $(P=0.026)$, and ASA score of more than II $(P<0.001)$ were determined to be the independent prognostic risk factors for postoperative complications. During the 30-day follow-up period, there was no postoperative mortality or readmission. Conclusions. Previous intestinal resection in patients with CD is an independent predictor of overall postoperative complications.
\end{abstract}

\section{Introduction}

Crohn's disease $(\mathrm{CD})$ is a chronic bowel inflammatory disease, mainly affecting the digestive tract, that frequently shows clinical symptoms in a relapsing and remitting manner [1]. The incidence of CD is higher in developed countries; however, with the development of its economy, China now is witnessing an increasing incidence of $\mathrm{CD}$ [2]. The aim of the therapeutic strategies is to maintain remission, with the purpose of preventing complications and disease progression.

Despite medical progress in the therapy for $\mathrm{CD}$, the surgical risk rate after 1,5 , and 10 years from the diagnosis was reported to be about $16 \%, 33 \%$, and $45 \%$, respectively [3]. According to reports on the natural history of the disease, more than half of the patients with $\mathrm{CD}$ will undergo more than one surgery in their lifetime [4]. Although surgery is an effective way to remove the intestinal lesions and relieve symptoms, surgical resection is not a curative method for CD. Postoperative complications are very common, including anastomotic leakage, fistula formation, bowel obstruction, intestinal bleeding, and surgical site infection (SSI) [5, 6]. The key medications applied in the current treatment of $\mathrm{CD}$, including immunomodulators, biologic drugs, and steroid hormones, are related to an increased incidence of postoperative complications after CD surgery $[7,8]$.

Preoperative treatment is necessary for patients with $\mathrm{CD}$, including drainage of abdominal abscesses or intestinal fistulas, provision of nutritional support, induction of remission of $\mathrm{CD}$, and use of biological agents [9]. Retrospective studies have tried to evaluate the risk factors for postoperative complications and have reported that advanced age, lower body mass index (BMI), poor nutritional status, higher American 
Society of Anesthesiologists (ASA) Physical Status Classification System score (ASA score), and the CD complications of abscess or intestinal fistula formation were related to increased postoperative complications [10-14]. Patients' overall condition has been improved through preoperative treatment and perioperative surgical home practice according to our previous research [15]. Because of the high CD recurrence rate and their complications, more than $50 \%$ of patients with $C D$ will experience surgical recurrence within 10 years after the first operation [16]. However, the specific impact of the previous intestinal resection on the postoperative complications in patients with $\mathrm{CD}$ was still uncertain. To evaluate the influence of previous intestinal resection on postoperative complications of $\mathrm{CD}$, we reviewed patients with $\mathrm{CD}$ who underwent surgery in our center. We also assessed the risk factors for postoperative complications in these patients.

\section{Materials and Methods}

2.1. Study Population. A cross-sectional study design was employed. The data of patients with CD were gathered from a retrospectively established database of a tertiary center from January 2016 through December 2019. Included in the current study were a total of 129 consecutive patients with CD who underwent surgical treatment at our Department of General Surgery. Patients with previous surgery for perianal disease, drainage of an abdominal abscess, or planned secondary surgery were excluded from this study. The study was approved by the Institutional Review Board of the Shanghai Ninth People's Hospital, Shanghai Jiao Tong University School of Medicine (Reference number: 2018297).

2.2. Clinical Assessment. Details from a prospective patient database comprising clinical data, including population demographics, preoperative case features, surgery-related characteristics, and postoperative outcome, were analyzed retrospectively. A standardized form was applied to acquire patient profiles, including detailed information on their illness and any surgery-related complications. Sex, age, BMI, and disease duration after the initial diagnosis of CD were all retrieved. Records were also collected regarding the disease characteristics, including Montreal classification for inflammatory bowel diseases, previous CD-related intestinal resection, and perianal lesions. The following surgeryrelated data were collected: ASA score, indications for surgery, intraoperative findings, estimated intraoperative blood loss, and estimated operative time. The type of procedure (open vs. conversion vs. laparoscopic approach) was also recorded. Preoperative medication records included the use of steroids, 5-aminosalicylic acid/sulfasalazine, immunomodulators, and biologic agents.

2.3. Assessment of Postoperative Complications. The primary endpoint of the study was the overall postoperative complications. Postoperative complications were defined as deviations that occurred from the routine postoperative procedure using the Clavien-Dindo classification system [17]. Specific 30-day postoperative complications including SSI, anasto- motic leak, urinary tract infection (UTI), and pneumonia were recorded. The occurrence of ileus was also noted; it was defined as abnormal postoperative bowel function recovery or the use of a nasogastric tube because of vomiting without evidence of mechanical bowel obstruction [18]. Postoperative small-bowel obstruction (SBO) was considered to be the existence of mechanical intestinal obstruction symptoms associated with supporting findings on imaging studies $[19,20]$. In addition, other parameters including dehydration, acute renal failure (ARF), and reoperation were noted. Hospital readmission was reviewed if it occurred within 30 days after discharge from the hospital.

2.4. Statistical Analysis. Continuous data were shown as mean \pm standard deviation (SD) and were analyzed by the independent Student $t$-test or Mann-Whitney $U$ test. The Kolmogorov-Smirnov test was used to test the normality of the data [21]. The categorical variables were reported as absolute frequencies and were analyzed by Pearson's chi-squared test or Fisher's exact test. Statistically significant factors by univariate analysis were further tested to find the independent factors related to postoperative complications by a multivariate logistic regression model. Two-tailed $P$ values $<0.05$ were considered to be statistically different. Statistical analyses were conducted with IBM SPSS Statistics for Windows, version 22.0 (IBM Corp., Armonk, New York, USA).

\section{Results}

3.1. Patient Demographics. A total of 129 patients with CD who underwent abdominal operations were included in the study; of these, $62(48.06 \%)$ patients had a previous bowel resection. Characteristics of the patients in the two groups are detailed in Table 1. The mean (SD) age of patients who had undergone previous resection by the time of surgery was 40.56 (9.65) years, older than the 37.12 (8.31) years of the patients who had not undergone previous resection $(P=0.031)$. The mean (SD) duration of $\mathrm{CD}$ from the time of diagnosis in the group that had undergone previous resection was $8.45(4.08)$ years, longer than the $6.85(3.89)$ years in the group having no previous resection $(P=0.025)$. The mean (SD) BMI of the previous resection group was 17.67 (1.07), lower than the 18.27 (1.14) in the group having no previous resection $(P=0.003)$. The main clinical manifestations of the disease were ileocolonic location and penetrating disease behavior, which were confirmed in about $45 \%$ of patients with $\mathrm{CD}$. The patients who had undergone previous resection were more likely to use less 5 -aminosalicylic acid/sulfasalazine preoperatively $(P=0.013)$. Patients with previous CD-related bowel resection were associated with an increased ASA score $(P=0.043)$. The two groups showed no statistically significant difference in terms of sex, disease behavior, disease location, perianal disease, or preoperative use of medications (biologics, steroids, or immunomodulators). Most of the patients underwent surgery mainly for bowel fistula that could have progressed from CD-related bowel disease such as abscess or stricture.

Table 2 shows the surgical variables of the two patient groups. The most common surgery conducted in the group 
TABle 1: Patient demographics and preoperative interventions.

\begin{tabular}{|c|c|c|c|}
\hline Characteristics & No previous resection $(n=67)$ & Previous resection $(n=62)$ & $P$ value \\
\hline Male, no. (\%) & $38(56.72)$ & $28(45.16)$ & 0.190 \\
\hline Age at surgery, mean (SD) $(y)$ & $37.12(8.31)$ & $40.56(9.65)$ & 0.031 \\
\hline BMI, mean (SD) & $18.27(1.14)$ & $17.67(1.07)$ & 0.003 \\
\hline Disease duration since diagnosis, mean (SD) (y) & $6.85(3.89)$ & $8.45(4.08)$ & 0.025 \\
\hline Disease behavior, no. (\%) & & & 0.708 \\
\hline B1: nonstricturing, nonpenetrating & $15(22.39)$ & $11(17.74)$ & \\
\hline B2: stricturing & $24(35.82)$ & $21(33.87)$ & \\
\hline B3: penetrating & $28(41.79)$ & $30(48.39)$ & \\
\hline Disease location, no. (\%) & & & 0.791 \\
\hline L1: terminal ileum & $23(34.33)$ & $19(30.65)$ & \\
\hline L2: colon & $15(22.39)$ & $13(20.97)$ & \\
\hline L3: ileocolon & $25(37.31)$ & $27(43.55)$ & \\
\hline L4: upper gastrointestinal tract & $4(5.97)$ & $3(4.84)$ & \\
\hline Perianal disease, no. (\%) & $14(20.90)$ & $17(27.42)$ & 0.386 \\
\hline \multicolumn{4}{|l|}{ Preoperative descriptions, no. (\%) } \\
\hline Biologics & $15(22.39)$ & $21(33.87)$ & 0.146 \\
\hline 5-ASA/sulfasalazine & $25(37.31)$ & $11(17.74)$ & 0.013 \\
\hline Steroids & $18(26.87)$ & $16(25.81)$ & 0.891 \\
\hline Immunomodulators & $14(20.90)$ & $17(27.42)$ & 0.386 \\
\hline Indications for surgery, no. (\%) & & & 0.945 \\
\hline Unresponsiveness to medical management & $13(19.40)$ & $11(17.74)$ & \\
\hline Bowel fistula(e) with or without disease-related abscess(es) & $26(38.81)$ & $24(38.71)$ & \\
\hline Fibrosis/stricturing & $23(34.33)$ & $23(37.10)$ & \\
\hline Other & $5(7.46)$ & $4(6.45)$ & \\
\hline ASA score, no. (\%) & & & 0.043 \\
\hline 1 & $17(25.37)$ & $9(14.52)$ & \\
\hline 2 & $36(53.73)$ & $27(43.55)$ & \\
\hline 3 & $12(17.91)$ & $22(35.48)$ & \\
\hline 4 & $2(2.99)$ & $4(6.45)$ & \\
\hline
\end{tabular}

BMI: body mass index; 5-ASA: 5-aminosalicylic acid; ASA: American Society of Anesthesiologists.

with no previous intestinal resection was ileocolonic resection, followed by small-bowel resection and segmental colectomy. However, the most common course in the previous resection group was small-bowel resection followed by ileocolonic resection and segmental colectomy $(P=0.026)$. Compared with the patients in the previous resection group, more patients with CD in the group with no previous resection underwent laparoscopic surgery $(P=0.031)$. The mean (SD) duration of operation in the previous resection group was $130.76(27.58) \mathrm{min}$, longer than that of $117.43(21.86)$ $\mathrm{min}$ in the group with no previous resection $(P=0.003)$. Patients with previous resection had a significantly greater estimated intraoperative blood loss than those without previous resection (mean [SD], 159.18 [59.06] mL vs. 129.33 [42.68] $\mathrm{mL} ; P=0.001)$. There were no statistical differences with regard to intraoperative findings of bowel fistulas and abscesses between the two groups.

3.2. Relationship between Previous Resection and Postoperative Complications. The postoperative complica- tions within 30 days compared between the two groups are shown in Table 3. There were no deaths or readmissions. The total postoperative complication rate of our cohort was 42.64\%. Patients of the previous resection group were associated with an increased rate of overall postoperative morbidity, especially anastomotic leak $(P=0.021)$ and severe postoperative complications (Clavien-Dindo grade III/IV) $(P=0.038)$. Meanwhile, there was no statistical significance in terms of SSI rate, UTI, pneumonia, SBO, ileus, dehydration, or ARF compared with patients without previous resection. Patients receiving previous resection had a statistically longer hospital stay than those without previous resection (mean [SD], 13.21 [6.68] days vs. 9.49 [4.06] days; $P<0.001)$.

3.3. Predictors of Postoperative Complications. In the current study, previous intestinal resection, increased ASA score, increased estimated blood loss, preoperative use of steroids, and longer hospital stay were all demonstrated to be associated with postoperative morbidity by univariate analysis 
TABLE 2: Surgical data.

\begin{tabular}{lcc}
\hline Characteristics & No previous resection $(n=67)$ & Previous resection $(n=62)$ \\
\hline Surgical procedures, no. (\%) & & $28(45.16)$ \\
$\quad$ Small-bowel resection & $17(25.37)$ & $14(22.58)$ \\
$\quad$ lleocolic resection & $30(44.78)$ & $12(19.35)$ \\
$\quad$ Segmental colectomy & $9(13.43)$ & $8(12.90)$ \\
$\quad$ Other (ostomy closure, strictureplasty, and stoma creation & $11(16.42)$ & $22(35.48)$ \\
without resection) & & $21(33.87)$ \\
Surgical technique, no. (\%) & $38(56.72)$ & $19(30.65)$ \\
$\quad$ Laparoscopy & $19(28.36)$ & 0.031 \\
$\quad$ Open surgery & $10(14.93)$ & $25(40.32)$ \\
$\quad$ Conversion & & $14(22.58)$ \\
Intraoperative findings, no. (\%) & $22(32.84)$ & $130.76(27.58)$ \\
$\quad$ Fistula(e) & $17(25.37)$ & $159.18(59.06)$ \\
$\quad$ Abscess(es) & $117.43(21.86)$ & 0.371 \\
Duration of operation, mean (SD) (min) & $129.33(42.68)$ & 0.003 \\
Estimated blood loss, mean (SD) (mL) & & 0.001 \\
\hline
\end{tabular}

TABle 3: Postoperative outcomes.

\begin{tabular}{|c|c|c|c|}
\hline Outcome & $\begin{array}{l}\text { No previous resection } \\
\qquad(n=67)\end{array}$ & $\begin{array}{c}\text { Previous resection } \\
\quad(n=62)\end{array}$ & $P$ value \\
\hline Overall complications, no. (\%) & $23(34.33)$ & $32(51.61)$ & 0.047 \\
\hline SSIs, no. (\%) & $13(19.40)$ & $16(2.58)$ & 0.384 \\
\hline Superficial incisional & $7(10.45)$ & $8(12.90)$ & 0.664 \\
\hline Deep incisional & $3(4.48)$ & $4(6.45)$ & 0.710 \\
\hline Organ or space infection & $3(4.48)$ & $4(6.45)$ & 0.710 \\
\hline Anastomotic leak, no. (\%) & $4(5.97)$ & $12(19.35)$ & 0.021 \\
\hline UTI, no. (\%) & $1(1.49)$ & $2(3.23)$ & 0.608 \\
\hline Pneumonia, no. (\%) & $2(2.99)$ & $3(4.84)$ & 0.671 \\
\hline SBO, no. (\%) & $6(8.96)$ & $9(14.52)$ & 0.325 \\
\hline Ileus, no. (\%) & $10(14.93)$ & $12(19.35)$ & 0.504 \\
\hline Dehydration, no. (\%) & $2(2.99)$ & $3(4.84)$ & 0.671 \\
\hline Acute renal failure, no. (\%) & $1(1.49)$ & $3(4.84)$ & 0.350 \\
\hline Severe postoperative complications (Clavien-Dindo grade III/IV), no. (\%) & $7(10.45)$ & $15(24.19)$ & 0.038 \\
\hline Length of hospital stay, mean (SD) (d) & $9.49(4.06)$ & $13.21(6.68)$ & $<0.001$ \\
\hline
\end{tabular}

SSI: surgical site infection; UTI: urinary tract infection; SBO: small-bowel obstruction.

(Table 4). Furthermore, three independent prognostic risk factors for postoperative complications were previous intestinal resection $(\mathrm{OR}=1.621 ; 95 \% \mathrm{CI}=1.017-2.453$; $P=0.019$ ), preoperative use of steroids ( $\mathrm{OR}=1.597 ; 95 \%$ $\mathrm{CI}=1.086-2.347 ; P=0.026)$, and ASA score of more than II $(\mathrm{OR}=1.854 ; 95 \% \mathrm{CI}=1.271-2.705 ; P<0.001)($ Table 5$)$.

\section{Discussion}

As far as we know, the current study is the first to evaluate the risk factors of complications after reoperation for patients with CD. Our results indicate that patients with CD who had undergone previous surgery were more inclined to be older and have the following: lower BMI, longer disease duration since diagnosis, less use of 5-aminosalicylic acid/sulfasalazine, and higher ASA score. The previous intestinal resection increased the risk of postoperative morbidity within 30 days for patients with CD after surgery, especially anastomotic leak and severe postoperative complications (Clavien-Dindo grade III/IV).

Despite great progress in the medical treatment for CD, accompanied by the sequential introduction of steroids, immunomodulators, and biologics, more than half of patients with $\mathrm{CD}$ eventually have to undergo surgical treatment over the course of the disease [22]. The repeat intestinal resection rate in our cohort was $48 \%$, which is similar to other studies showing a $25-45 \%$ rate of repeat resection [23]. The short-term outcomes after surgery for CD have 
TABLE 4: Risk factors associated with postoperative complications.

\begin{tabular}{|c|c|c|c|}
\hline Variables & $\begin{array}{l}\text { Unremarkable } \\
\text { postoperative } \\
\text { course }(n=74)\end{array}$ & $\begin{array}{c}\text { Any postoperative } \\
\text { complications }(n=55)\end{array}$ & $P$ value \\
\hline \multicolumn{4}{|l|}{ Univariate analysis } \\
\hline Previous intestinal resection for CD, no. (\%) & $29(39.19)$ & $33(60.00)$ & 0.019 \\
\hline Male, no. (\%) & $36(48.65)$ & $30(54.55)$ & 0.508 \\
\hline Age at surgery, mean $(\mathrm{SD})(\mathrm{y})$ & $38.90(9.96)$ & $38.60(7.90)$ & 0.851 \\
\hline BMI, mean (SD) & $18.10(1.20)$ & $17.82(1.06)$ & 0.171 \\
\hline Disease duration since diagnosis, mean (SD) $(y)$ & $7.37(4.45)$ & $7.94(3.45)$ & 0.433 \\
\hline Disease behavior, no. (\%) & & & 0.712 \\
\hline B1: nonstricturing, nonpenetrating & $14(18.92)$ & $12(21.82)$ & \\
\hline B2: stricturing & $28(37.84)$ & $17(30.91)$ & \\
\hline B3: penetrating & $32(43.24)$ & $26(47.27)$ & \\
\hline Disease location, no. (\%) & & & 0.211 \\
\hline L1: terminal ileum & $22(29.73)$ & $20(36.36)$ & \\
\hline L2: colon & $13(17.57)$ & $15(27.27)$ & \\
\hline L3: ileocolon & $34(45.95)$ & $18(32.73)$ & \\
\hline L4: upper gastrointestinal tract & $5(6.76)$ & $2(3.64)$ & \\
\hline Perianal disease, no. (\%) & $16(21.62)$ & $15(27.27)$ & 0.458 \\
\hline \multicolumn{4}{|l|}{ Preoperative descriptions, no. (\%) } \\
\hline Biologics & $17(22.97)$ & $19(34.55)$ & 0.147 \\
\hline 5-ASA/sulfasalazine & $16(21.62)$ & $20(36.36)$ & 0.065 \\
\hline Steroids & $14(18.92)$ & $20(36.36)$ & 0.026 \\
\hline Immunomodulators & $16(21.62)$ & $15(27.27)$ & 0.458 \\
\hline Indications for surgery, no. (\%) & & & 0.302 \\
\hline Unresponsiveness to medical management & $10(13.51)$ & $14(25.45)$ & \\
\hline Bowel fistula(e) with or without disease-related abscess(es) & $32(43.24)$ & $18(32.73)$ & \\
\hline Fibrosis/stricturing & $26(35.14)$ & $20(36.36)$ & \\
\hline Other & $6(8.11)$ & $3(5.45)$ & \\
\hline ASA score, no. (\%) & & & 0.003 \\
\hline 1 & $20(27.03)$ & $6(10.91)$ & \\
\hline 2 & $39(52.70)$ & $24(43.64)$ & \\
\hline 3 & $12(16.22)$ & $22(40.00)$ & \\
\hline 4 & $3(4.05)$ & $3(5.45)$ & \\
\hline Surgical procedures, no. (\%) & & & 0.259 \\
\hline Small-bowel resection & $27(36.49)$ & $18(32.73)$ & \\
\hline Ileocolic resection & $29(39.19)$ & $15(27.27)$ & \\
\hline Segmental colectomy & $9(12.16)$ & $12(21.82)$ & \\
\hline Other (ostomy closure, strictureplasty, stoma creation without resection) & $9(12.16)$ & $10(18.18)$ & \\
\hline Surgical technique, no. (\%) & & & 0.139 \\
\hline Laparoscopy & $39(52.70)$ & $21(38.18)$ & \\
\hline Open surgery & $18(24.32)$ & $22(40.00)$ & \\
\hline Conversion & $17(22.97)$ & $12(21.82)$ & \\
\hline \multicolumn{4}{|l|}{ Intraoperative findings, no. (\%) } \\
\hline Fistula $(e)$ & $23(31.08)$ & $24(43.64)$ & 0.143 \\
\hline Abscess(es) & $15(20.27)$ & $16(29.09)$ & 0.246 \\
\hline Duration of operation, mean (SD) (min) & $121.72(26.04)$ & $126.69(24.85)$ & 0.276 \\
\hline Estimated blood loss, mean (SD) (mL) & $133.85(43.49)$ & $156.89(61.90)$ & 0.014 \\
\hline Length of hospital stay, mean (SD) (d) & $9.34(2.87)$ & $13.88(7.46)$ & $<0.001$ \\
\hline
\end{tabular}

CD: Crohn's disease; BMI: body mass index; 5-ASA: 5-aminosalicylic acid: ASA: American Society of Anesthesiologists. 
TABLE 5: Multivariate analysis of risk factors for postoperative complications.

\begin{tabular}{lcc}
\hline Variables & $\begin{array}{c}\text { Odds ratio } \\
(95 \% \mathrm{CI})\end{array}$ & $P$ value \\
\hline Estimated blood loss & $1.407(1.005-1.969)$ & 0.081 \\
Steroids & $1.597(1.086-2.347)$ & 0.026 \\
Previous intestinal resection for CD & $1.621(1.071-2.453)$ & 0.019 \\
ASA score & $1.854(1.271-2.705)$ & $<0.001$ \\
\hline
\end{tabular}

CD: Crohn's disease; CI: confidence interval; ASA: American Society of Anesthesiologists.

been markedly improved by better perioperative treatment. However, postoperative morbidity still occurs in up to $40 \%$ of patients after surgery [24]. The current study emphasizes that repeat intestinal resection is associated with a significant increase in postoperative complications, particularly anastomotic leak. Patients with $\mathrm{CD}$ undergoing initial resection had a $5 \%$ incidence of anastomotic leak, similar to the rates published previously $[25,26]$. However, repeat resection dramatically increased the leak rate to $19 \%$ compared with patients undergoing initial resection, leading to a threefold increase in anastomotic leak rates. The findings of this study support previous resection as a risk factor for anastomotic leak in patients with $\mathrm{CD}$. Repeat resection may be an alternative marker for a more aggressive disease form, more complicated surgery, or altered vascular distribution. Repeat resection may suggest a prolonged dissection because of the atypical planes of bowel dissection, leading to increased risk of accidental enterotomy and additional bowel devascularization. Meanwhile, our study has also shown that operative time and the length of hospital stay of patients with $\mathrm{CD}$ undergoing primary intestinal resection were shorter than for those who underwent reoperation. Estimated blood loss appeared to be smaller in the group with no previous resection. The mortality and readmission rates were zero.

Studies have reported that preoperative antitumor necrosis factor therapy, preoperative steroids, preoperative poor nutritional status, recurrent $\mathrm{CD}$, immunosuppressant medications, and the perforating $\mathrm{CD}$ phenotype were identified as risk factors for postoperative complications in CD [12, $27,28]$. In this study, we tried to determine the risk factors associated with postoperative morbidity. For overall complications, using univariate analysis in addition to previous intestinal resection, preoperative corticosteroids, higher ASA score, and greater estimated blood loss were also related to an increased rate of postoperative morbidity. Furthermore, previous intestinal resection, preoperative use of steroids, and ASA score higher than II were determined to be independent predictors of postoperative complications by multivariate analysis.

We acknowledge that this study has certain limitations. First, our current study was not designed as randomized control research and only included patients from a single center. All the patients who were enrolled were surgical patients who might have had greater disease severity, and the patients who had undergone previous intestinal resection might be more prevalent in our center. Therefore, there may be selection bias. Second, preoperative medication use was determined from medical records in our department. Although we did our best to carefully scrutinize all the available medication records, there may be bias. Finally, laboratory studies, including preoperative level of C-reactive protein, erythrocyte sedimentation rate, and albumin level, which might have a significant impact on postoperative complications, were also not analyzed in this study. Additional prospective clinical studies with more patients should be performed to further evaluate the results of our current study.

\section{Conclusions}

In conclusion, the current study has demonstrated that previous intestinal resection in patients with $\mathrm{CD}$ is a risk factor for postoperative complications. Furthermore, this study revealed that previous intestinal resection particularly increased the risk for anastomotic leak and severe postoperative complications. The findings should be incorporated into future surgical decision-making, especially with respect to the indications of intestinal resection and anastomosis.

\section{Data Availability}

The datasets used in the current study are available from the corresponding author on reasonable request.

\section{Ethical Approval}

The Institutional Review Board of Shanghai Ninth People's Hospital, Shanghai Jiao Tong University School of Medicine, approved the project (Reference number: 2018297).

\section{Conflicts of Interest}

The authors have no conflicts of interest to declare.

\section{Acknowledgments}

We thank all our patients for their participation in this study and the staff at the Department of General Surgery, Shanghai Ninth People's Hospital, Shanghai Jiao Tong University School of Medicine. This study was supported by the National Natural Science Foundation of China (No. 81802303); Ethicon "Excellence in Surgery" Grant (HZB20181119-9); the Project of Biobank (YBKB-201916) from Shanghai Ninth People's Hospital, Shanghai Jiao Tong University School of Medicine; Clinical Research Program of Ninth People's Hospital, Shanghai Jiao Tong University School of Medicine (JYLJ022); Clinical Research MDT Program of Ninth People's Hospital, Shanghai Jiao Tong University School of Medicine (201701008); and Research Project of Health and Family Planning Commission of Pudong New Area (PW2018D-01).

\section{References}

[1] D. C. Baumgart and W. J. Sandborn, "Crohn's disease," Lancet, vol. 380 , no. 9853 , pp. 1590-1605, 2012. 
[2] J. Zhao, S. C. Ng, Y. Lei et al., "First prospective, populationbased inflammatory bowel disease incidence study in mainland of China: the emergence of "western" disease," Inflammatory Bowel Diseases, vol. 19, no. 9, pp. 1839-1845, 2013.

[3] A. D. Frolkis, J. Dykeman, M. E. Negrón et al., "Risk of surgery for inflammatory bowel diseases has decreased over time: a systematic review and meta-analysis of population-based studies," Gastroenterology, vol. 145, no. 5, pp. 996-1006, 2013.

[4] S. C. Ng, W. K. Leung, H. Y. Shi et al., "Epidemiology of inflammatory bowel disease from 1981 to 2014: results from a territory-wide population-based registry in Hong Kong," Inflammatory Bowel Diseases, vol. 22, no. 8, pp. 1954-1960, 2016.

[5] T. Yamamoto, R. N. Allan, and M. R. B. Keighley, "Risk factors for intra-abdominal sepsis after surgery in Crohn's disease," Diseases of the Colon \& Rectum, vol. 43, no. 8, pp. 1141$1145,2000$.

[6] T. Fontana, N. Falco, M. Torchia, R. Tutino, and G. Gulotta, "Bowel perforation in Crohn's disease: correlation between CDAI and Clavien-Dindo scores," Giornale di Chirurgia, vol. 38, no. 6, pp. 303-312, 2017.

[7] F. N. Aberra, J. D. Lewis, D. Hass, J. L. Rombeau, B. Osborne, and G. R. Lichtenstein, "Corticosteroids and immunomodulators: postoperative infectious complication risk in inflammatory bowel disease patients," Gastroenterology, vol. 125, no. 2, pp. 320-327, 2003.

[8] U. Ahmed Ali, S. T. Martin, A. D. Rao, and R. P. Kiran, "Impact of preoperative immunosuppressive agents on postoperative outcomes in Crohn's disease," Diseases of the Colon \& Rectum, vol. 57, no. 5, pp. 663-674, 2014.

[9] P. Zerbib, D. Koriche, S. Truant et al., "Pre-operative management is associated with low rate of post-operative morbidity in penetrating Crohn's disease," Alimentary Pharmacology \& Therapeutics, vol. 32, no. 3, pp. 459-465, 2010.

[10] A. Kanazawa, T. Yamana, K. Okamoto, and R. Sahara, "Risk factors for postoperative intra-abdominal septic complications after bowel resection in patients with Crohn's disease," Diseases of the Colon \& Rectum, vol. 55, no. 9, pp. 957-962, 2012.

[11] A. Alves, Y. Panis, Y. Bouhnik, M. Pocard, E. Vicaut, and P. Valleur, "Risk factors for intra-abdominal septic complications after a first ileocecal resection for Crohn's disease: a multivariate analysis in 161 consecutive patients," Diseases of the Colon \& Rectum, vol. 50, no. 3, pp. 331-336, 2007.

[12] A. Brouquet, L. Maggiori, P. Zerbib et al., “Anti-TNF therapy is associated with an increased risk of postoperative morbidity after surgery for ileocolonic Crohn disease: results of a prospective nationwide cohort," Annals of Surgery, vol. 267, no. 2, pp. 221-228, 2018.

[13] S. Argeny, A. Stift, M. Mittlböck et al., "Advanced age impacts surgical characteristics and postoperative course in patients with Crohn's disease," International Journal of Surgery, vol. 33, no. Part A, pp. 182-186, 2016.

[14] V. Celentano, D. P. O’Leary, A. Caiazzo et al., "Longer small bowel segments are resected in emergency surgery for ileocaecal Crohn's disease with a higher ileostomy and complication rate," Techniques in Coloproctology, vol. 23, no. 11, pp. 10851091, 2019.

[15] H. Xu, D. Yao, Y. Huang, H. Fan, and Y. Li, "Perioperative surgical home model improves outcomes in Crohn's disease patients undergoing disease-related surgery," Gastroenterology Research and Practice, vol. 2020, Article ID 4293420, 7 pages, 2020.
[16] L. Guizzetti, G. Zou, R. Khanna et al., "Development of clinical prediction models for surgery and complications in Crohn's disease," Journal of Crohn's and Colitis, vol. 12, no. 2, pp. 167-177, 2018.

[17] D. Dindo, N. Demartines, and P. A. Clavien, "Classification of surgical complications: a new proposal with evaluation in a cohort of 6336 patients and results of a survey," Annals of Surgery, vol. 240, no. 2, pp. 205-213, 2004.

[18] J. Gu, L. Stocchi, F. H. Remzi, and R. P. Kiran, “Total abdominal colectomy for severe ulcerative colitis: does the laparoscopic approach really have benefit?," Surgical Endoscopy, vol. 28, no. 2, pp. 617-625, 2014.

[19] N. Goussous, K. M. Kemp, M. P. Bannon et al., "Early postoperative small bowel obstruction: open vs laparoscopic," American Journal of Surgery, vol. 209, no. 2, pp. 385-390, 2015.

[20] S. B. S. Sajja and M. Schein, "Early postoperative small bowel obstruction," British Journal of Surgery, vol. 91, no. 6, pp. 683-691, 2004.

[21] P. Mishra, C. M. Pandey, U. Singh, A. Gupta, C. Sahu, and A. Keshri, "Descriptive statistics and normality tests for statistical data," Annals of Cardiac Anaesthesia, vol. 22, no. 1, pp. 67-72, 2019.

[22] S. Panteleimonitis, J. Ahmed, T. Parker, T. Qureshi, and A. Parvaiz, "Laparoscopic resection for primary and recurrent Crohn's disease: a case series of over 100 consecutive cases," International Journal of Surgery, vol. 47, pp. 69-76, 2017.

[23] O. Bernell, A. Lapidus, and G. Hellers, "Risk factors for surgery and postoperative recurrence in Crohn's disease," Annals of Surgery, vol. 231, no. 1, pp. 38-45, 2000.

[24] M. Fumery, P. Seksik, C. Auzolle et al., "Postoperative complications after ileocecal resection in Crohn's disease: a prospective study from the REMIND group," American Journal of Gastroenterology, vol. 112, no. 2, pp. 337-345, 2017.

[25] J. A. Waters, T. Francone, P. W. Marcello et al., "Quality improvement initiatives in colorectal Surgery," Diseases of the Colon \& Rectum, vol. 60, no. 2, pp. 213-218, 2017.

[26] C. Galata, C. Weiss, J. Hardt et al., "Risk factors for early postoperative complications and length of hospital stay in ileocecal resection and right hemicolectomy for Crohn's disease: a single-center experience," International Journal of Colorectal Disease, vol. 33, no. 7, pp. 937-945, 2018.

[27] W. Huang, Y. Tang, L. Nong, and Y. Sun, "Risk factors for postoperative intra-abdominal septic complications after surgery in Crohn's disease: a meta-analysis of observational studies," Journal of Crohn's and Colitis, vol. 9, no. 3, pp. 293-301, 2015.

[28] M. Abou Khalil, J. Abou-Khalil, J. Motter et al., "Immunosuppressed patients with Crohn's disease are at increased risk of postoperative complications: results from the ACS-NSQIP database," Journal of Gastrointestinal Surgery, vol. 23, no. 6, pp. 1188-1197, 2019. 